\title{
Making Drug Discovery More Efficient Applying Statistical Entropy to Biology
}

\author{
Philip D. Houck \\ Department of Medicine (Division of Cardiology) Baylor Scott \& White Health, Temple, TX, USA \\ Email: Philip.Houck@bswhealth.org
}

How to cite this paper: Houck, P.D. (2020) Making Drug Discovery More Efficient Applying Statistical Entropy to Biology. Journal of Modern Physics, 11, 1969-1976. https://doi.org/10.4236/jmp.2020.1112124

Received: November 18, 2020 Accepted: December 15, 2020 Published: December 18, 2020

Copyright (c) 2020 by author(s) and Scientific Research Publishing Inc. This work is licensed under the Creative Commons Attribution International License (CC BY 4.0).

http://creativecommons.org/licenses/by/4.0/ (c) (i) Open Access

\begin{abstract}
Biology without governing principle makes predications impossible. Observations lead to some successful therapies, and to unexpected failures. Erwin Schrödinger attempted to quantify biology with the concept of negative entropy. These insights lead to fundamental principles of biologic entropy. The quantification of negative entropy is difficult to calculate since the number of parts of the body and the way these parts are arranged is very large (atomistic disorder). There can be approximations that answer questions such as why females live longer, and why a lower body temperature predicts longevity. This concept can reveal the culprit of diabetes II; understanding the microbiome can reduce its entropy by increasing the entropy of its host. The real advantage of statistical entropy is finding new drugs and predicting viral mutations based on energetics and negative entropy. The misfolding of a protein will increase the entropy of an individual with the result of early death. The calculations of biologic entropy require the knowledge of each developmental step, and the statistical possibilities of the next step. If the step is crucial to maintain low entropy, a carrier protein will assure the energetics of the step is favorable. This protein is the target of new therapies.
\end{abstract}

\section{Keywords}

Negative Entropy, Quantification of Biology, Carrier Proteins, Drug Development

\section{Introduction}

Erwin Schrödinger was a famous early $20^{\text {th }}$ century physicist who first proposed the concept of negative entropy to explain the ordering of life. He also conjectured the existence of a memory molecule that preserves life information from one generation to the next. Erwin Schrödinger (1944), "What Is Life? The Phys- 
ical Aspect of the Living Cell". The excerpt below is based on lectures delivered under the auspices of the Dublin Institute for Advanced Studies at Trinity College, Dublin, in February 1943.

"How would we express in terms of the statistical theory the marvelous faculty of a living organism, by which it delays the decay into thermodynamical equilibrium (death)? We said before: 'It feeds upon negative entropy', attracting, as it were, a stream of negative entropy upon itself, to compensate the entropy increase it produces by living and thus to maintain itself on a stationary and fairly low entropy level. If $\mathrm{D}$ is a measure of disorder, its reciprocal, $1 / \mathrm{D}$, can be regarded as a direct measure of order. Since the logarithm of 1/D is just minus the logarithm of $\mathrm{D}$, we can write Boltzmann's equation thus:

$$
-(\text { entropy })=k \log (1 / D) \text {. }
$$

Hence the awkward expression 'negative entropy' can be replaced by a better one: entropy, taken with the negative sign, is itself a measure of order. Thus, the device by which an organism maintains itself stationary at a fairly high level of the orderliness (=fairly low level of entropy) really consists continually sucking orderliness from its environment. This conclusion is less paradoxical than it appears at first sight. Rather could it be blamed for triviality? Indeed, in the case of higher animals we know the kind of orderliness they feed upon well enough, viz. the extremely well-ordered state of matter in more or less complicated organic compounds, which serve them as foodstuffs. After utilizing it they return it in a very much degraded form, not entirely degraded, however, for plants can still make use of it (These, of course, have their most power supply of 'negative entropy' the sunlight).

Entropy $=k \log D$, where $k$ is the so-called Boltzmann's constant $=(3.2983 \times$ $10^{24} \mathrm{cal} . / \mathrm{C}$ ), and $D$ a quantitative measure of the atomistic disorder of the body in question [1]".

The above introduction by Erwin Schrödinger states best the difference between life and physical properties of the universe. Attempts to justify statistical entropy as a force of biology; explaining evolution and other difficult biological questions are explored in the paper "Should negative entropy be included in the fundamental laws of biology?" [2]. The key to understanding negative entropy in biology is the determination of atomistic disorder, $D$.

$D$ is the sum of all the parts and all the possible ways the parts fit together. The human body has 50 trillion cells of roughly 300 cell types. The human also has 500 trillion bacteria and even more viruses and fungi in the microbiome inhabiting the gut, skin, airway, and every organ system. In addition, each cell has the entire information to make another individual with the molecule predicted by Schrödinger identified as Deoxyribonucleic acid DNA. In Men there are 46 chromosomes and in women there are 45 with one of the $\mathrm{X}$ chromosomes blocked, since it is a duplicate. Four base pairs describe 20,000 genes that encode 2 million proteins. Separate structures called mitochondria may have been parasitic bacteria that now live within our cells symbiotically. The proteins consisting 
of 20 amino acids build cell organelles surface markers messenger RNA and the means to communicate between all our cells. The structure of proteins and the folding of branch chains is a complex entropic question adding significantly to atomistic disorder. $\mathrm{D}$ is a very big number [2]. Computations must be approximated. A huge number of small equations need to be solved to find the source of environmental energy.

The equation is useful and can give insight to mysteries that perplex biological scientist. Why do women live longer than men? The first question was addressed in the paper "Why is there a young woman advantage? Why is it lost? Applying the Laws of Biology to Men and Women" [3]. Men are more complex having 46 chromosomes as opposed to 45 in women. Men live less; approximated (1 $45 / 46) \times$ average death age of females. Table 1 lists some principles of biologic entropy.

Why does a low body temperature predict a longer life? Negative entropy is temperature dependent and humans have an approximate temperature of 310 $\mathrm{K}^{\circ}$. A lower body temperature suggests a lower state of entropy. Entropy is not time dependent but is temperature dependent [4]. The lower the temperature the less entropy.

Biologic age seems to be time dependent since it is observed that old people are closer to death. Older individuals have had more processes occur than younger individuals. The processes are time dependent. After a process is completed, statistical possibilities will be available for the next step. The number of possibilities is the atomistic disorder. The favored completed process is the lowest energy requiring state. In biology there is ordering of disorder taking energy from the environment. Biologic age is reduced by efficiently accepting energy from the environment. Other pathways could be statistically chosen and represents noise in a biological process. Entropy is not reduced and biologic age increases pushing patients to an early death. If the process is critical to survival, there will

Table 1. Fundamental principles of biologic entropy.

- Health is defined as maintaining low entropy

- Disease is a failure to maintain low entropy

- Entropy, introducing changes in DNA, favors evolution so life can survive in a changing environment

- Fetal development is an ordering of substrates and requires negative entropy with the energy supplied by the environment

- Repair of Injury depends on the developmental state. Prior to birth, injury is directed toward complete functional repair (negative entropy). After birth, repair is directed toward rapid return of partial function at the expense of achieving complete repair (positive entropy)

- Age is a state of increasing entropy

- Biologic processes are time dependent ordered by information provided by DNA. At the end of each process there are statistical possibilities for the next step. The energy required for this step is negative entropy $=k^{*}$ LOG $(1 / D)$.

- A strategy that saves energy by supplying a catalyst, carrier protein, or electromagnetic force assures the next critical step has the lowest energy.

- Drug discovery finds the catalyst, carrier protein, or electromagnetic force that favors negative entropy 
be a mechanism that lowers the energy requirement to assure a successful process. One mechanism of securing the correct pathway is carrier proteins that can reduce the energy required to complete the critical step. These carrier proteins are targets for new drug discoveries.

Information science and negative entropy is a way of quantifying biological systems making systems biology more predictable. Each pathway in systems biology has a probabilistic outcome based the total possible interactions for the next step. An additional carrier protein may or not be present to assure the outcome if the desired outcome requires more energy. Quantification can be added to the multiple arrows and diagrams required in system analysis. Quantification can suggest a missing element that could serve as a drug target.

\section{Current Pharmacological Short Falls}

Pharmacology had not kept pace with new discoveries in biology. Pharmacological principles are mired in the receptor hypothesis and pharmacokinetics. The search is for protein and receptor matches, downstream effects and regulators of the response. Twenty thousand genes provide codes to make two million proteins. Researchers are invested in their proteins and tease out mechanisms of actions hoping their protein is the solution to human suffering. These targets of new therapies are elusive, often found serendipitously or teased out of simple analysis of single cells or simple organisms. A newer approach of systems biology is intensely complex and involves pathways within multiple organelles and interaction between cells. This approach also leads to new drug discoveries in a painfully slow manner. Escape pathways, inhibitors, and alternate stimulations/inhibitions lead to unexpected failures. The proteins are often abandoned. The more information obtained the more complex systems biology becomes. Since proteins are encoded by similar genes, they can have the same basic structure and can influence another pathway that was totally unsuspected. The drug industry is flourishing with a new technique of modifying receptors or proteins by antibodies to upregulate or down regulate pathways. The development identifies, purifies, elicits properties, and fulfills the 5 steps of drug approval. The estimated cost per drug approaches 3 Billion dollars [5]. The cost of identifying all 2 million proteins is a Quadrillion $\left(10^{15}\right) \$$ (Table 2).

Once the drug has come to market, the clinician finds new attributes of the drug not predicted by pharmacokinetics or systems biology. An example of new attributes is HMG CoA Reductase Inhibitors (Statins) that lower cholesterol but also modifies the immune system and increases circulating stem cells [6] [7]. The latter discovered effects are more important in decreasing myocardial infarction,

Table 2. Steps in the development of drugs.

\footnotetext{
1) Discovery and development

2) Pre-clinical Research

3) Clinical Research

4) FDA Review

5) FDA Post-Market Safety Monitoring
} 
surviving infections, reducing perioperative complications, and repair of the fatty liver despite warning of its use with liver enzyme elevation. Pleomorphic benefits are attributed to properties of 3-hydroxy-3-methylglutaryl-coenzyme (HMG-CoA) reductase inhibitors. The pleomorphic effects escaped the basic scientist who only considered this molecule in lipid metabolism. Statins appear to have a negative entropy affect. The mechanism of interaction with the immune system that promotes repair over destruction is still not known. The property that reduces inflammation is observed and not understood. The answer is complex requiring knowledge the interaction of the drug with all the parts of the body. When energy of the desired outcome exceeds the energy of entropy a catalyst, carrier protein, or electromagnetic force is required to assure success, repair over decay. Statins provide this function favoring repair over decay. Table 3 proposes why current methods of drug discovery are inefficient.

\section{Example of Entropy and Disease}

Diabetes II is a modern disease first described in 1936 by Dr. Himsworth. He describes a disease of elevated glucose with excess insulin [10]. The disease is very commonly associated with abdominal obesity, elevated blood pressure, atherogenic dyslipidemia, insulin resistance manifested as glucose intolerance, and pro-inflammatory, and pro-thrombotic states. The cause of the disease is not elucidated. Entropy should be suspected. The greatest source of entropy is the virome followed by the microbiome. The changing diet of excess calories, carbohydrates, modern invention of agriculture; favors the microbiome as the strongest entropic energy in this disease. Glucose and carbohydrates are easy for bacteria to consume and process [11]. Taking this energy from the environment lowers the microbiome's entropy. It is to the bacteria's favor to elevate glucose in

Table 3. Criticism of current techniques.

1) There are no fundamental laws of biology used to model health and disease. Biology, lingering in observation, is not a science [8].

2) There is only one Cell. Subspecialty silos partition cardiovascular drugs, cancer therapies, and diabetes drugs failing to acknowledge that there is only one cell. Therefore, drugs in one discipline may have used in other disciplines that are undiscovered.

3) Biological system noise is dismissed. Carrier proteins that in themselves have no biologic activity can allow proteins to fold in a preferred manner permitting function, partially block a receptor, or the misfolded proteins lead to a serious disease [9].

4) Using surrogate risk factors for biological measured defects is inefficient and does not account for individual compensation. Biological biomarker measures should select patients for drug therapies to increase the efficiency of studies. Some biologic markers are pivotal representing a major shift in biologic balance identifying disease and treatment of disease.

5) Inflammation is a term explaining all chronic disease states. Inflammation can increase or decrease entropy. The state of the immune system that perpetuates these states is not measured. Knowledge to characterize immune system malfunction is necessary.

6) Drugs in normal subjects may not behave the same as in diseased states due to disease activation of genes that are not present in normal subjects.

7) The study of energetics in protein folding, molecular interactions, and influence of the electromagnetic environment on protein formation is in its infancy, complex, and poorly organized. 
the host. If the bacteria make a protein that partially blocks the insulin receptor or attaches to insulin making it less likely to fit in to the receptor; glucose will become more abundant since the human cells are unable to process the glucose. This simple protein lowers the microbiome entropy and raises the human entropy. Finding the protein and neutralizing its effects represents a new therapy. Entropy supports more research in the microbiome to solve the disease state of diabetes II. Observational medicine is slowly getting to the same conclusion but painfully slow. Table 4 taken from "Should negative entropy be included in the fundamental laws of biology?" [2] demonstrates other diseases of entropy.

\section{Roadmap to the Future}

Biology must be quantified. Calculation of energy of every sequence of embryological development from two cells to an entire organism. The entropic energy for two cells to become one is $3.67 \times 10^{-23}$ cal supplied by the carbohydrate in semen. The subsystems of DNA replication, gene activation, protein production must be quantified in all the possible outcomes. The energy requirement and noted discrepancies between entropy and the energy available from the environment are calculated. New drugs will be found to explain the discrepancies. Once the energies and processes are described the origin of life could be modeled.

Unfortunately, the number of permutations and calculations to describe every step in the development of a human would take to the end of our sun. The good news is once a calculation is performed it does not have to be performed again.

Table 4. Diseases of entropy and interventions.

\begin{tabular}{cccc}
\hline Disease/Affliction & Component of life & Defect Promoting Increased Entropy & Entropy Intervention \\
\hline Age & Chromosomes & Failure to copy DNA correctly & Increase Telomere length \\
Age & Cells & Decreased stem cells & Increase stem cells \\
Age/red cell aplasia & Types of Cells & Lack of red cell precursor & Replace Red Cell Precursor \\
$\begin{array}{c}\text { Age/Hutchinson-Gilford's } \\
\text { progeroid syndrome }\end{array}$ & Genes & mutation in LMNA & Replace Gene \\
Evolution & Base pairs & Point mutation & Depending on the environment this \\
Alzheimer's Dementia & Protein Folding & Abnormally folded amyloid beta protein & Inhibit protein productionRefold \\
miss-folded protein
\end{tabular}


Piecemeal calculations can give insight and useful information. The largest contributor to biologic entropy is the virus which is relatively small and can be potentially studied so that every step of its production can be sequenced, and energy of that step calculated. The virus is subject to mutations and these mutations could be predicted understanding the energetics of its development. Vaccines could be produced prior to the mutation so that endemics could be halted.

Information science and negative entropy is a way of quantifying biological systems making systems biology more predictable. Each pathway in systems biology has a probabilistic outcome based on all the possible interaction for the next step. An additional carrier protein may or not be present to assure the outcome if the desired outcome requires more energy. Quantification can be added to the multiple arrows and diagrams required in system analysis. Quantification can suggest a missing element that could serve as a drug target.

\section{New Physics from Biology}

Erwin Schrödinger was interested in life to discover new physics. He was fascinated how life preserved order at least for a fraction of time. The universe appears to have order for a greater fraction of time, and the energy to preserve this order is under intense investigation. Physicists have invented dark matter and dark energy to explain the orderly motion of stars in galaxies. Erwin Schrödinger, a physicist gave insight to biologist helping them understand a property of life negative entropy. As a cardiologist I would like to imagine how the Universe maintains order. Perhaps, space time has a thickness, a Planck length where light can travel as wave and emerge as a particle. Particles can emerge from either side of space time and be entangled over space while still being connected in space time. Cosmologists should look within Space time to find the energy that explains the ordering of galaxies. Imagine writing relativistic equation including space time thickness. Singularities are no longer a mathematical construct just a very large number.

\section{Conclusion}

Life is complicated. Biology lacks fundamental principles not allowing predictive models. Erwin Schrödinger pondered upon this weakness of biology and proposed negative entropy as a means of quantifying biologic processes. He did not provide the solution only the path forward. The solution is not trivial and requires energetics to every protein twist, bonds between receptors and proteins. The processes of life from intercellular protein production to cell differentiation, assembly, and repair needs catalogued. Successful sequencing of DNA over the last 50 years pales to the task of quantifying life. Drugs will still be discovered serendipitously, but energetics can be applied to specific disease processes such as amyloid related dementia, a disease of protein mal formation. Processes lowering entropy can extend life and reduce disease burden. Entropic forces point to the microbiome as an important predictor of health and disease. Age 
represents a failure to maintain low entropy. Determining the failure to lower entropy as we age will be new drug targets. The concept of negative entropy was not well received in the lectures by Erwin Schrödinger; but, as he said "entropy, taken with the negative sign, is itself a measure of order." New physics and understanding the order of the Universe will arise from application of the second law of thermodynamics statistical entropy.

\section{Conflicts of Interest}

The author declares no conflicts of interest regarding the publication of this paper.

\section{References}

[1] Erwin, S. (1944) What Is Life?: The Physical Aspect of the Living Cell. Based on Lectures Delivered under the Auspices of the Dublin Institute for Advanced Studies at Trinity College, Dublin, in February 1943. Chapter 6 Order, Disorder and Entropy.

[2] Houck, P.D. (2014) OA Biology, 2, 7.

[3] Houck, P.D. (2014) JCVD, 10, 1-8.

[4] Ben-Naim, A. (2020) Entropy, 22, 430. https://doi.org/10.3390/e22040430

[5] Wouters, O.J., McKee, M. and Luyten, J. (2020) JAMA, 323, 844-853. https://doi.org/10.1001/jama.2020.1166

[6] Ridker, P., Danielson, E., Fonseca, F., et al. (2009) The Lancet, 373, 1175-1182. https://doi.org/10.1016/S0140-6736(09)60447-5

[7] Vasa, M., Fichtlscherer, S., Adler, K., et al. (2001) Circulation, 103, 2885-2890. https://doi.org/10.1161/hc2401.092816

[8] Houck, P.D. (2015) Medical Hypotheses, 85, 266-271. https://doi.org/10.1016/j.mehy.2015.05.011

[9] Argyrou, A. (2020) The Misfolding of Proteins. In: Vlamos, P., Ed., GeNeDis 2018, Advances in Experimental Medicine and Biology, Vol. 1195, Springer, Cham, 249-254. https://doi.org/10.1007/978-3-030-32633-3 33

[10] Himsworth, H.P. (1936) Lancet, I, 127-130. https://doi.org/10.1016/S0140-6736(01)36134-2

[11] Houck, P.D. and de Oliveira, J.M.F. (2013) Medical Hypotheses, 80, 637-642. https://doi.org/10.1016/j.mehy.2012.11.019 